Localizador: 19063 doi: 10.35366/93977

\title{
Evaluación de calidad de vida en pacientes con acné: validación de una escala de medición
}

\author{
Evaluation of quality of life in patients with acne: validation of a measurement scale \\ Sandra Muvdi,* Socorro Moreno, ${ }^{\ddagger}$ Viviana Rodríguez, ${ }^{\S}$ Laura Charry॥
}

Palabras clave:

Calidad de vida

relacionada con la

salud, acné, validación

lingüística, validación

psicométrica.

Keywords:

Health related quality of

life, acne, linguistic

validation, psychometric

validation.

* Médica Dermatóloga, MsC Epidemiología Clínica, Hospital Universitario Centro Dermatológico «Federico Lleras Acosta». Bogotá, Colombia. ORCID 00000002-8530-2109.

₹ Psicóloga, MsC Epidemiología Clínica, Profesor asistente, Departamento de Epidemiología Clínica y Bioestadística. Pontificia Universidad Javeriana. ORCID 0000-0002-41194409.

§stadística, $\mathrm{MsC}$

Epidemiología Clínica, Profesor asistente, Departamento de Epidemiología Clínica y Bioestadística. Pontificia Universidad Javeriana. ORCID 0000-0001-84870676.

\section{Recibido:}

21/Noviembre/2019.

Aceptado:

19/Enero/2020.

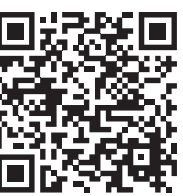

\section{Resumen}

Introducción: En Colombia no existen instrumentos específicos validados para evaluar la calidad de vida con acné. Este estudio realizó validación lingüística al español y psicométrica de un instrumento para evaluar la calidad de vida con acné. Material y métodos: Validación psicométrica en pacientes con acné. La consistencia interna y la estabilidad en el tiempo se evaluaron con coeficientes $\alpha$ de Cronbach y correlación de Lin, mientras que la sensibilidad al cambio fue calculada con respuesta media estandarizada (SMR) y la evidencia relacionada con contenido con el método Delphi. Análisis factorial para evaluar evidencia relacionada con estructura interna. La evidencia de validez de constructo se evaluó con prueba de Kruskal Wallis o correlación de Spearman. Resultados: La consistencia interna es adecuada ( $\alpha$ Cronbach 0.74-0.90); la estabilidad moderada (la correlación de Lin fue de 0.67-0.72). La sensibilidad al cambio satisfactoria (SMR 0.61-0.90) y los resultados de asociaciones con otras variables constituyen evidencia de validez de constructo. El análisis factorial confirmatorio permite concluir que la estructura de cuatro dominios no se mantiene en esta versión y el análisis factorial exploratorio de factores principales propone una estructura de tres factores. Conclusiones: La versión en español de la escala Acne-QoL se considera equivalente conceptual y semánticamente a la versión original, demuestra adecuadas propiedades psicométricas, pero puede beneficiarse de modificaciones.

\section{ABStRACT}

Introduction: In Colombia there are no specific validated instruments to assess quality of life in acne. This study performed a Spanish and psychometric linguistic validation of an instrument to assess quality of life in acne. Material and methods: Psychometric validation in patients with acne. Internal consistency and stability over time were evaluated with Cronbach's $\alpha$ and Lin correlation coefficients, sensitivity to change by calculating standardized mean response (SMR) and content related evidence with Delphi method. Factor analysis was performed to evaluate internal structure. Evidence of construct validity was evaluated with Kruskal Wallis or Spearman correlation. Results: Internal consistency is adequate (Cronbach's $\alpha$ of 0.74-0.90); stability is moderate (Lin's correlation 0.67 to 0.72 ). The sensitivity to change presents satisfactory results (SMR $0.61-0.90)$ and the results of associations with other variables provide evidence of construct validity. The confirmatory factor analysis allows to conclude that the four domains structure is not maintained and the exploratory factor analysis using principal factor analysis yields three factors. Conclusions: The Spanish version of the Acne-QoL scale is considered conceptually and semantically equivalent to the original version, demonstrating adequate psychometric properties, but can benefit from some modifications.

\section{INTRODUCCIÓN}

E I acné es una enfermedad crónica, autolimitada y de fácil diagnóstico que afecta aproximadamente a $80 \%$ de la población, especialmente a adolescentes y a adultos jóvenes, ${ }^{1}$ e impacta su calidad de vida. ${ }^{2}$ Algunos estudios han mostrado que las personas con acné tienen una baja calidad de vida y un mayor riesgo de trastornos del estado del ánimo o de ansiedad..$^{3-5}$ Sin embargo, la severidad del acné no siempre se relaciona de manera directa con el impacto en la calidad de vida; ${ }^{6-10}$ por lo tanto, medir el impacto de la enfermedad en la calidad de vida permitiría optimizar el proceso de toma de decisiones, orientar de una mejor manera los tratamientos de los pacientes y evaluar la efectividad de los mismos. ${ }^{3}$

Aunque existen escalas globales y dermatológicas que se han utilizado para evaluar la calidad de vida en pacientes con acné, ${ }^{11-18}$ en Colombia no existen instrumentos específicos validados disponibles. Este estudio tiene como objetivo realizar la adaptación cultural (validación lingüística y psicométrica) de un instrumento para evaluar la calidad de vida relacionada con la salud ( $\mathrm{CVrS}$ ) en pacientes con acné. Éste fue seleccionado tras evaluar 
" Médica Dermatóloga, MsC Epidemiología Clínica, Profesor Instructor, Unidad de Dermatología. Facultad de Medicina. Pontificia Universidad Javeriana, Hospital Universitario San Ignacio. ORCID 0000-0002 4457-6824

Conflicto de intereses: Ninguno. los principales instrumentos disponibles en la literatura, siguiendo un proceso metodológico de acuerdo con las recomendaciones del ISPOR Task Force for Translation and Cultural Adaptation y del MAPI Research Institute y la aplicación correspondiente para la evaluación de características psicométricas a partir de la evidencia combinada de diferentes fuentes.

\section{MATERIAL Y MÉTODOS}

\section{Selección y validación lingüística del instrumento}

Para identificar los instrumentos específicos de acné se realizó una búsqueda en la base de datos bibliográfica MEDLINE de la Biblioteca Nacional de Medicina de los Estados Unidos, utilizando los términos de búsqueda: «Acne Vulgaris» [Mesh], «Quality of Life» [Mesh] y «Psychometrics» [Mesh]. La versión original de los instrumentos identificados y los artículos relacionados con los mismos fueron evaluados con el instrumento EMPRO ${ }^{19}$ y los criterios de revisión desarrollados por Scientific Advisory Committee of the Medical Outcomes Trust (SAC). ${ }^{20}$ A través de este proceso se identificó el «Quality of Life Questionnaire: Acne-QoL» como la mejor escala disponible, debido al método de desarrollo y los resultados de pruebas psicométricas. La escala Acne-QoL es un cuestionario autoadministrado desarrollado para caracterizar el impacto del acné facial en pacientes con acné leve, moderado y severo entre 13 y 35 años, evalúa a través de 19 preguntas temas que se pueden agrupar en cuatro dominios: autopercepción, rol social, rol emocional y síntomas. ${ }^{21-23}$

La validación lingüística se realizó siguiendo los pasos recomendados por ISPOR Task Force for Translation and Cultural Adaptation ${ }^{21}$ y los procedimientos propuestos por el MAPI Research Institute. ${ }^{22}$ Las fases desarrolladas con el fin de obtener equivalencia semántica y cultural se presentan en la Figura 1.

Durante el proceso de aclaración cognoscitiva se identificaron dos preguntas con dificultades en la interpretación: ítem 1 (sentirse poco atractivo) y 14 (interacción con personas del sexo opuesto o del mismo si es homosexual), por lo que fue necesario realizar ajustes. El ítem 1 requirió invertir el sentido de la pregunta, de negativo a positivo (sentirse atractivo). En el ítem 14 se decidió preguntar sobre las personas que le atraen (sin especificar la preferencia sexual). Al final de este proceso se obtuvo la versión en español para Colombia de la escala Acne-QoL con una equivalencia semántica, conceptual y cultural con la versión original en inglés.

\section{Validación psicométrica}

\section{Participantes}

Se incluyeron pacientes entre 12 y 35 años con diagnóstico de acné facial primario que consultaron al Hospital Universitario Centro Dermatológico «Federico Lleras Acosta» (CDFLLA) de Bogotá, Colombia. Se contó con aprobación del protocolo por el Comité de Investigaciones y Ética de la Facultad de Medicina de la Pontificia Universidad Javeriana y el Comité Científico del CDFLLA y se contó con la financiación del CDFLLA para el desarrollo del mismo. Se excluyeron pacientes en manejo con corticoides, terapia de reemplazo hormonal, vitamina B12, antiepilépticos, tuberculostáticos, psicofármacos, pacientes con alguna incapacidad física o mental que impidiera responder adecuadamente los instrumentos, así como analfabetas, pacientes que no aceptaran participar o que no firmaron el consentimiento informado.

El tamaño de muestra se basó en las recomendaciones de Nunnally y Comrey y Lee, ${ }^{24,25}$ quienes proponen al menos 300 sujetos. Para evaluar la confiabilidad test-retest y la sensibilidad al cambio, considerando un error tipo I de 0.05, un coeficiente de correlación en la población de al menos 0.8 y una amplitud de $10 \%$, a dos colas, se calculó un tamaño de muestra de 150 pacientes.

\section{Procedimiento de recolección}

El proceso de recolección se dividió en tres visitas. En la primera se diligenció el formato de recolección que incluía variables demográficas, las preguntas de la versión en español de la escala Acne-QoL y de las subescalas del tamizaje de ansiedad y depresión (Hospital Anxiety and Depression Scale, versión validada para Co- 
lombia), ${ }^{23,26-28}$ la calificación de la satisfacción con la vida y el estado de salud y la evaluación de la apariencia física (escala Measure of Body Apperception [MBA] ${ }^{29}$ validada en español. Los sujetos se citaron siete días después de la primera visita para diligenciar el Acne-QoL, asumiendo que no habría cambios en la medición. El formulario fue enviado por correo electrónico a los pacientes que no podían asistir. Los participantes diligenciaron una vez más el Acne-QoL dos o tres meses después de la primera visita (recomendación de consenso de dermatólogos para observar respuesta terapéutica). Con el fin de optimizar la adherencia, se ofreció una consulta de control gratuita con el dermatólogo en la tercera visita a los pacientes que diligenciaran los tres formularios.
Paso o proceso

Proceso de toma de decisiones

\section{Desenlaces}

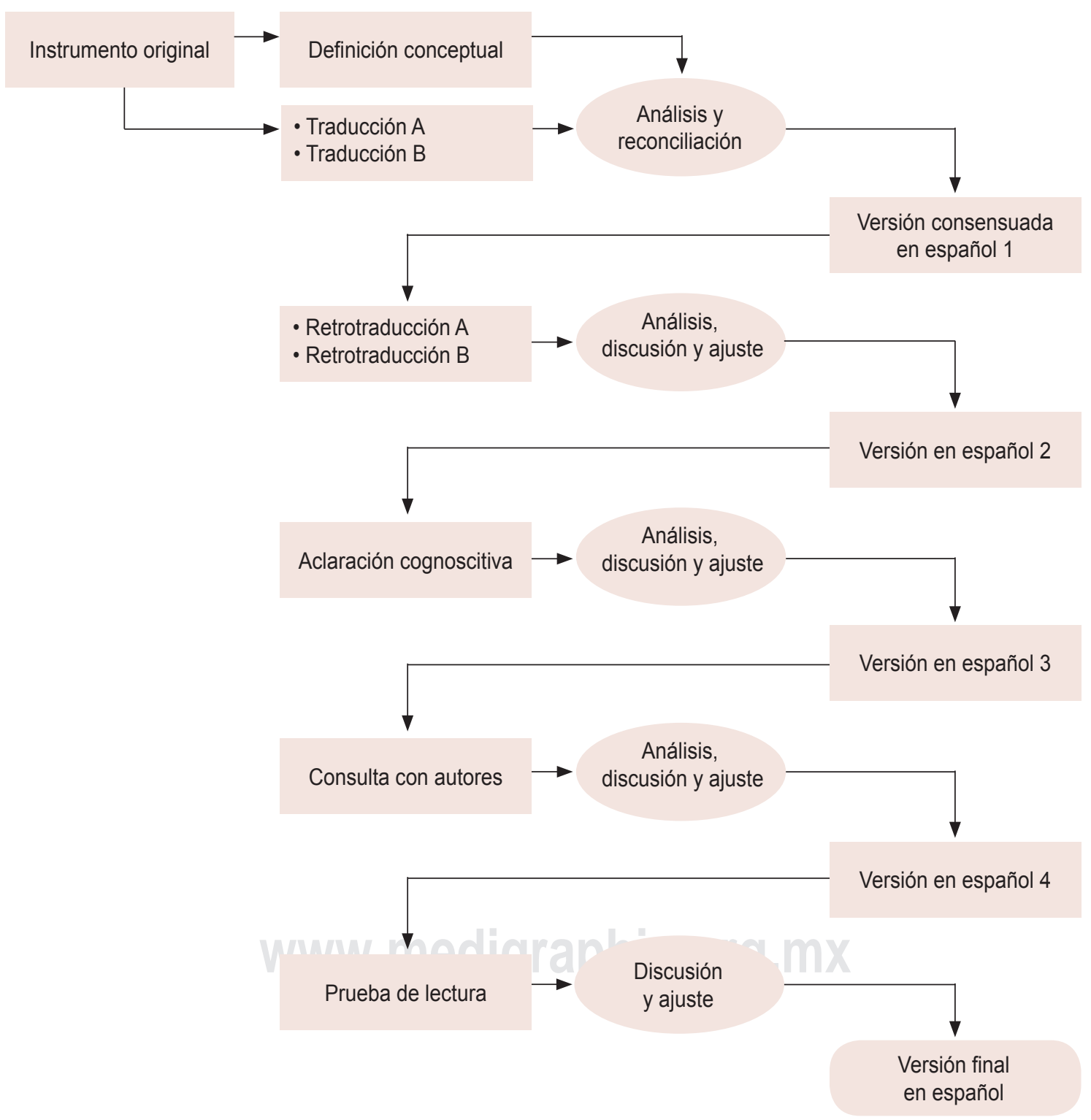

Figura 1: Proceso de traducción y adaptación lingüística y cultural al español colombiano. 
Tabla 1: Descripción de métodos utilizados para la validación psicométrica.

\begin{tabular}{|c|c|c|}
\hline Fase & Propósito & Metodología \\
\hline $\begin{array}{l}\text { Descripción de la } \\
\text { población }\end{array}$ & Evaluación del contexto & $\begin{array}{l}\text { Análisis descriptivo de las características de la muestra utilizando la mejor medida de } \\
\text { resumen según cada variable }\end{array}$ \\
\hline \multirow[t]{2}{*}{ Consistencia interna } & Correlación entre ítems & - $\alpha$ de Cronbach por dominios \\
\hline & $\begin{array}{l}\text { Evaluar beneficio de eliminar uno } \\
\text { o más ítems }\end{array}$ & - Cambios en $\alpha$ de Cronbach eliminando ítems \\
\hline Confiabilidad test-retest & $\begin{array}{l}\text { Evaluación de la estabilidad en el } \\
\text { tiempo }\end{array}$ & $\begin{array}{l}\text { Coeficiente de correlación de Lin (variable mostró evidencia de no seguir una distri- } \\
\text { bución normal) }\end{array}$ \\
\hline Sensibilidad al cambio & $\begin{array}{l}\text { Evaluación del cambio en el tiempo } \\
\text { o por efecto del tratamiento }\end{array}$ & $\begin{array}{l}\text { - Tamaño del efecto estandarizado. Tamaño del efecto mayores a } 0.5 \text { fueron conside- } \\
\text { rados efectos importantes } 30,31 \\
\text { - Respuesta media estandarizada }\end{array}$ \\
\hline \multirow[t]{3}{*}{ Estimación de la validez } & $\begin{array}{l}\text { Evidencia relacionada con el } \\
\text { contenido }\end{array}$ & $\begin{array}{l}\text { - Evaluación de expertos: método Delphi } \\
\text { Evaluación de relevancia, comprensión, claridad, carga emocional, inducción de } \\
\text { respuesta y contextualización }\end{array}$ \\
\hline & $\begin{array}{l}\text { Evidencia relacionada con la } \\
\text { estructura interna }\end{array}$ & $\begin{array}{l}\text { - Análisis factorial confirmatorio. Evaluación del ajuste del modelo }\left(\chi^{2} \text {, promedio de }\right. \\
\text { los residuales estandarizados [RMSEA]) } \\
\text { - Análisis factorial exploratorio } \\
\text { Factores principales. Rotación oblicua Oblimin y Promax. Selección dentro de cada } \\
\text { factor los ítems con cargas mayores de } 0.5\end{array}$ \\
\hline & $\begin{array}{l}\text { Evidencia relacionada con la rela- } \\
\text { ción con otras variables }\end{array}$ & $\begin{array}{l}\text { - Medición de variables (con las que se consideró el constructo de CVrS en pacientes } \\
\text { con acné se encuentra teóricamente relacionado). Medidas con instrumentos inde- } \\
\text { pendientes: (ansiedad y depresión: escala Hospital Anxiety and Depression Sca- } \\
\text { le, } \text {, }^{23,26} \text { satisfacción con la vida y con el estado de salud: escala diferencial semántica } \\
\text { de } 7 \text { puntos) y la evaluación de la apariencia física: cuatro preguntas de la escala } \\
\text { Measure of Body Apperception (MBA) } \\
\text { - Empleando un enfoque basado en argumentos para la validez, }{ }^{37} \text { se plantearon } \\
\text { hipótesis que permitieran evaluar la asociación entre el Acne-QoL con las variables } \\
\text { seleccionadas: } \\
\text { - Hipótesis 1. A mayor puntaje autopercepción, menor severidad del acné } \\
\text { - Hipótesis 2. A mayor puntaje en autopercepción y en síntomas de acné, mayor } \\
\text { puntaje en dependencia de la imagen corporal } \\
\text { - Hipótesis 3. Sujeto clasificado como no caso de ansiedad o depresión, mayor } \\
\text { puntaje en rol emocional } \\
\text { - Hipótesis 4. Un puntaje alto en el rol emocional se relaciona con baja satisfac- } \\
\text { ción con la vida; sin embargo, el puntaje del rol emocional debería ser indepen- } \\
\text { diente de la satisfacción con el estado de salud } \\
\text { - Prueba de Kruskal-Wallis ajustado por múltiples comparaciones } \\
\text { Coeficiente de Spearman }\end{array}$ \\
\hline
\end{tabular}

\section{Análisis estadístico}

Se realizó la descripción de los participantes y de los puntajes de los dominios de la escala. La Tabla 1 describe los métodos utilizados en el proceso de validación psicométrica.

La consistencia interna se evaluó a través del coeficiente $\alpha$ de Cronbach usando los datos del Acne-QoL recolectados en la primera visita, lo cual permitió estudiar la correlación entre los ítems de la escala y el beneficio de eliminar alguno de ellos. La estabilidad en el tiempo se evaluó usando el coeficiente de correlación de Lin entre las mediciones del Acne-QoL en la primera y segunda visita. La sensibilidad al cambio (en el tiempo o por efecto de tratamiento) se evaluó calculando el tamaño del efecto estandarizado y la respuesta media estandarizada entre las mediciones del Acne-QoL en la primera y tercera visita.

La estimación de la validez se trianguló recolectando evidencia a través de diferentes procesos. La evidencia relacionada con el contenido fue evaluada con un método Delphi de expertos, que evaluó si el contenido del instrumento representaba el concepto para el cual fue diseñado. La evidencia relacionada con la estructura interna se inició con un análisis factorial confirmatorio y dado que no hubo ajuste del modelo se realizó análisis factorial exploratorio.

Se plantearon varias hipótesis que permitieran evaluar la asociación del Acne-QoL y las otras escalas recolectadas: 
Hospital Anxiety and Depression Scale (HADS), Satisfacción con la vida y el estado de salud y Measure of Body Apperception (MBA). Cuando las hipótesis comparaban el puntaje del Acne-QoL entre grupos se empleó la prueba de Kruskal-Wallis ajustando por múltiples comparaciones, y cuando dichas hipótesis correspondían a variables continuas o de intervalo se calculó el coeficiente de correlación de Spearman con su respectivo intervalo de confianza. Un nivel de error del 5\%, o menor en el caso del ajuste, se consideró significativo. Los análisis fueron realizados en STATA $12{ }^{\circledR}$.

\section{RESULTADOS}

Durante cuatro meses del año 2012 se invitó a participar a 388 personas que asistieron a consulta médica dermatológica en el CDFLLA, de las cuales 349 (89.9\%) cumplieron los criterios de selección. La Figura 2 muestra el número de pacientes incluidos en cada uno de los tres momentos de aplicación del instrumento y las razones principales de exclusión.

La edad promedio fue de 19.6 años (DE 4.6), la mayoría de los participantes eran solteros (91.1\%), 58.4\% fueron hombres, el tipo de acné más frecuente fue noduloquístico (50.1\%) y la mayoría fueron clasificados por el dermatólogo como acné moderado y severo (77.9\%). Las características demográficas y clínicas de los participantes en la primera aplicación se resumen en la Tabla 2.

De los 349 participantes, sólo uno (0.3\%) presentó un dato faltante en la escala en la pregunta «įQué tantos granos con pus tenía en su cara?» El puntaje de esta pregunta se imputó empleando la media del dominio, de acuerdo con las instrucciones del manual de aplicación.

El dominio con puntaje promedio más alto fue «autopercepción» (21.0 \pm 6.8) y el más bajo fue «síntomas de acné» (17.0 \pm 4.9$)$. La puntuación obtenida en cada uno de los cuatro dominios de la escala en la primera etapa se presenta en la Tabla 3.

\section{Evaluación de la consistencia interna}

La consistencia interna para cada uno de los dominios se consideró satisfactoria. El dominio con el $\alpha$ de Cronbach más bajo fue «síntomas de acné» mientras que el dominio con mayor consistencia interna fue «rol social». La Tabla 4 muestra los coeficientes de $\alpha$ de Cronbach por cada dominio y los resultados del cambio al eliminar cada uno de los ítems. Se evidencia que «autopercepción» (al eliminar «atractivo») y «síntomas de acné» (al eliminar «grasosa la piel») se benefician de la eliminación de algún ítem (puntajes más altos en el dominio al eliminar dicho ítem).

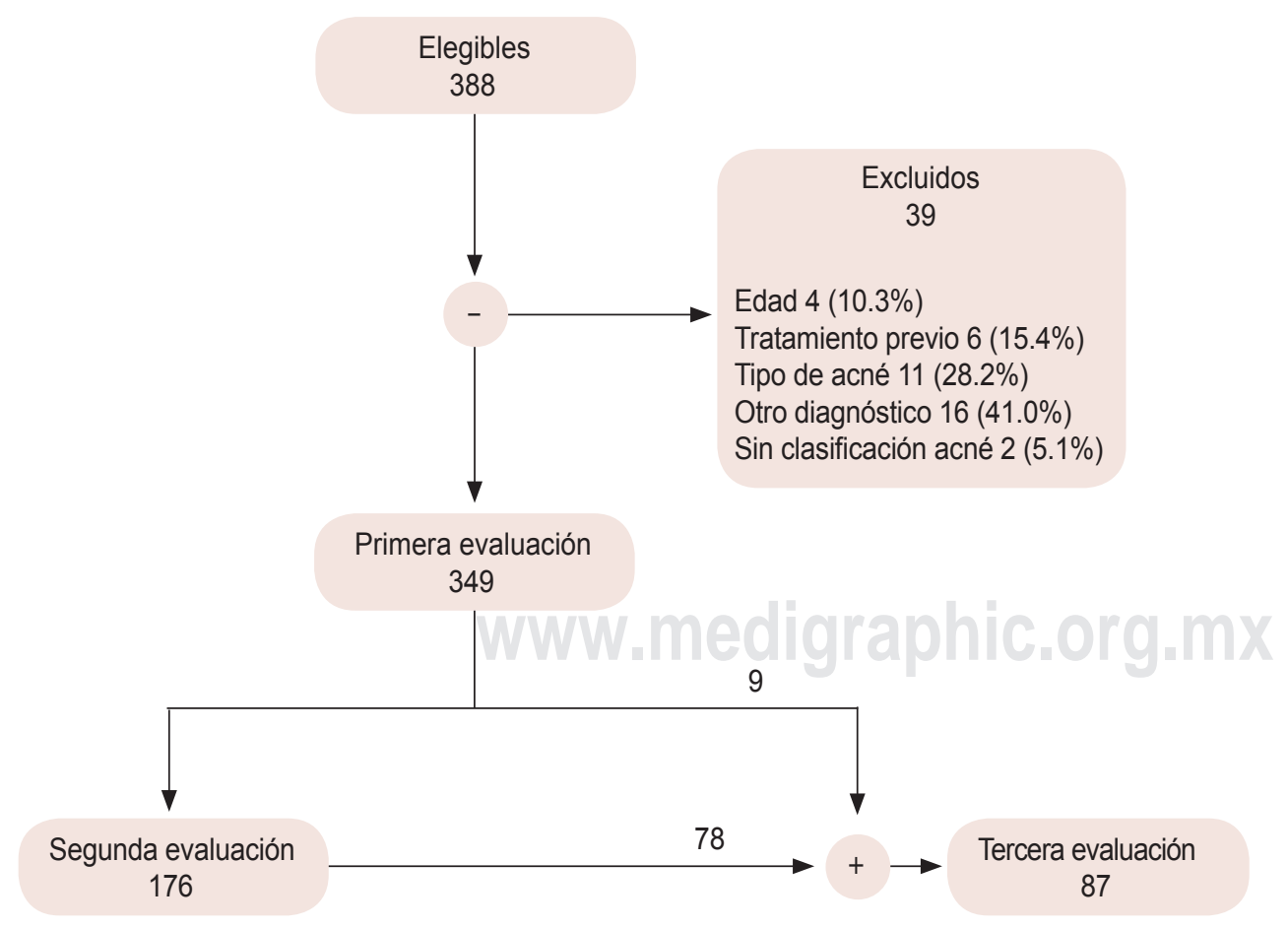

Figura 2:

Flujograma de pacientes. 


\begin{tabular}{|c|c|c|}
\hline Variable & $\mathrm{n}$ & $\%$ \\
\hline \multicolumn{3}{|l|}{ Sexo } \\
\hline Femenino & 145 & 41.55 \\
\hline Masculino & 204 & 58.45 \\
\hline \multicolumn{3}{|l|}{ Estado civil } \\
\hline Soltero & 318 & 91.12 \\
\hline Casado/unión libre & 27 & 7.74 \\
\hline Separado & 4 & 1.15 \\
\hline \multicolumn{3}{|l|}{ Nivel educativo } \\
\hline Primaria & 8 & 2.29 \\
\hline Bachillerato & 201 & 57.59 \\
\hline Técnico & 61 & 17.48 \\
\hline Profesional & 70 & 20.06 \\
\hline Postgrado & 9 & 2.58 \\
\hline \multicolumn{3}{|l|}{ Edad (años) } \\
\hline Media \pm DE & $19.56 \pm 4.56$ & \\
\hline Rango & 12-35 & \\
\hline Moda & 15 & \\
\hline \multicolumn{3}{|l|}{ Tipo de acné } \\
\hline Vulgar & 103 & 29.51 \\
\hline Noduloquístico & 175 & 50.14 \\
\hline Conglobata & 71 & 20.34 \\
\hline \multicolumn{3}{|c|}{ Severidad del acné según el paciente } \\
\hline Leve & 45 & 12.89 \\
\hline Moderado & 149 & 42.69 \\
\hline Severo & 125 & 35.82 \\
\hline Muy severo & 30 & 8.60 \\
\hline \multicolumn{3}{|c|}{ Severidad del acné de acuerdo con el médico dermatólogo } \\
\hline Leve & 77 & 22.06 \\
\hline Moderado & 186 & 53.30 \\
\hline Severo & 86 & 24.64 \\
\hline \multicolumn{3}{|c|}{ Severidad del acné según la escala GAE } \\
\hline Limpio & 4 & 1.15 \\
\hline Casi limpio & 21 & 6.02 \\
\hline Leve & 95 & 27.22 \\
\hline Moderado & 156 & 44.70 \\
\hline Severo & 65 & 18.62 \\
\hline Muy severo & 8 & 2.29 \\
\hline
\end{tabular}

\section{Evaluación de la estabilidad}

La estabilidad fue evaluada en una muestra de 176 participantes que completaron el instrumento en papel o a través de un formulario enviado por correo electrónico. El resultado de los coeficientes de correlación de Lin fue considerado como moderado para cada uno de los dominios de la escala (Tabla 3).

\section{Evidencia basada en relación con otras variables}

De acuerdo con las subescalas de tamizaje de ansiedad y depresión (Hospital Anxiety and Depression Scale), ${ }^{23,26}$
$65.9 \%$ de los pacientes fueron clasificados como no casos de ansiedad, $20.3 \%$ como casos dudosos y $13.8 \%$ como casos de ansiedad; mientras que para depresión, 91.1\% fueron clasificados como no casos, $6.9 \%$ como casos dudosos y $2.0 \%$ como casos. La distribución de frecuencias de la satisfacción con la vida y el estado de salud son muy similares, lo cual indica un porcentaje importante de satisfacción (Figura 3).

Se observaron diferencias en el puntaje del dominio «autopercepción» del Acne-QoL entre las categorías de severidad del acné evaluada por el paciente $(p<0.0001)$, con puntajes más altos a menor severidad del acné (severidad leve: $26.2 \pm 5.9$, severidad moderada: $23.1 \pm 5.6$ y severidad alta: $17.4 \pm 6.2$ ).

Los puntajes del dominio «emocional» fueron menores en casos de ansiedad (13.4 \pm 5.8$)$ que en los no casos (21.9 $\pm 5.9)(p=0.0001)$. Se observaron resultados similares en depresión, con menores puntajes en los casos de depresión $(15.3 \pm 7.1)$ que en los no casos (20.4 \pm 7.5$)(p=0.0008)$.

El coeficiente de Spearman entre «autopercepción» y evaluación de la imagen corporal fue de 0.45 (IC 95\% 0.37 a 0.54), mientras que entre «síntomas de acné» y la evaluación de la imagen corporal fue de 0.29 (IC 95\% 0.19 a 0.38). Al comparar los puntajes de «rol emocional» y los puntajes de la satisfacción con la vida y la satisfacción con el estado de salud, los coeficientes de Spearman fueron de -0.25 (IC 95\% -0.35 a -0.15) y -0.21 (IC 95\% -0.31 a -0.11), respectivamente.

\section{Evidencia basada en la estructura interna (análisis factorial)}

Se realizó un análisis factorial confirmatorio para cada uno de los cuatro dominios y no hubo un adecuado ajuste del modelo $\left(\chi^{2} 599.30\right.$ y RMSEA 0.093, grados de libertad 150, $p=0.0000$ ). Dado que había una alta correlación entre las variables según el índice de adecuación muestral KMO (Kaiser Meyer Olkin $=0.94)$ y la prueba de esfericidad de Bartlett ( $p<0.001)$, se realizó un análisis factorial exploratorio con el objetivo de examinar la estructura factorial de la versión en español para Colombia, utilizando el método de factores principales. Tres factores explicaban el 99.55\% de la varianza. Debido a que los factores no son independientes, se realizó rotación oblicua, tanto Oblimin como Promax con tres factores, y se seleccionaron dentro de cada factor los ítems con cargas mayores de 0.5. Al comparar estas rotaciones se encontró una misma composición, cargas similares, pero en la rotación Promax existía un ítem adicional, que de acuerdo con los resultados del análisis de consistencia interna no debería ser eliminado; por lo tanto, se decidió adoptar los resultados obtenidos con la rotación Promax. La estructura final de la escala se 
presenta en la Figura 4, en la que se muestran las cargas factoriales de cada ítem dentro de cada factor. El primer factor agrupaba la mayoría de los ítems de los dominios identificados originalmente como «autopercepción» y «rol social» (ítem 2, 3, 6, 10, 11, 12, 13 y 14). El segundo factor agrupaba los ítems del dominio identificado originalmente como «rol emocional» (ítem 4, 5, 8 y 9) y el tercer factor agrupaba los ítems del dominio identificado originalmente como «síntomas» (ítem 15, 16 y 17). Los ítems eliminados de la estructura factorial fueron cuatro (ítem 1, 7, 18 y 19).

\section{Evaluación de la sensibilidad al cambio}

Se realizó la tercera recolección de datos de la escala en 87 pacientes. Cambios en el tamaño del efecto mayores a 0.5 fueron considerados efectos importantes. ${ }^{30,31} \mathrm{El}$ dominio con el cambio más importante fue «síntomas de acné», seguido por «autopercepción» y «rol emocional»; y por último, «rol social» (Tabla 5).

\section{DISCUSIÓN}

Este estudio identificó la «Quality of Life Questionnaire: Acne QoL» como un instrumento idóneo dado que su proceso de desarrollo fue metodológicamente apropiado y presentaba adecuadas características psicométricas. ${ }^{32-34}$

Los retos para alcanzar la equivalencia semántica y evitar el sesgo de constructo proveniente de la propiedad diferencial de comportamientos relevantes para el constructo $^{35}$ implicaron la revisión conceptual de adjetivos que resultan unívocos en inglés y hallar conceptos en español verdaderamente equivalentes o ajustar la direccionalidad de los ítems que no resultaban comprensibles en su forma lingüísticamente equivalente.

Las propiedades psicométricas de la versión en español de la Acne-QoL fueron evaluadas en pacientes

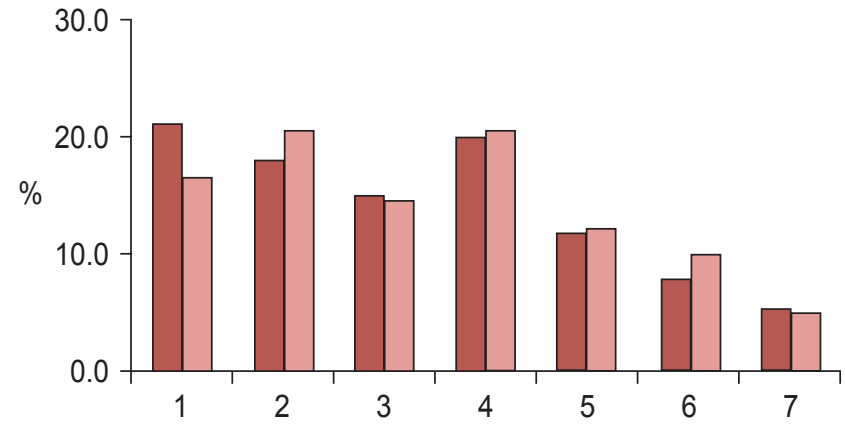

Muy satisfecho

Satisfacción con la vida

Satisfacción con el estado de salud

Figura 3: Satisfacción con la vida y el estado de salud.

con acné primario de diferentes tipos y severidades y en el presente estudio han mostrado ser satisfactorias. Las características demográficas y clínicas de los pacientes fueron similares al estudio de validación de la versión original de la escala Acne-QoL. ${ }^{33}$

La consistencia interna es adecuada y similar a la versión original de la escala Acne-QoL ${ }^{33,34}$ y el grado de concordancia entre los resultados obtenidos entre las diferentes aplicaciones es moderado, siendo el dominio de «síntomas de acné» el puntaje que menos estabilidad presentó. Es probable que esto de deba a que las lesiones clínicas de la enfermedad pueden variar muy rápidamente, pero es posible que el impacto general de la enfermedad sea valorado por los pacientes de una manera más global, por lo que la consistencia interna es mejor en los otros tres dominios. La consistencia interna reportada en la validación lingüística y cultural en francés ${ }^{36}$ es similar a la obtenida por nosotros; sin embargo, el estudio que evaluó la consistencia interna de la versión original en inglés reportó concordancias excelentes

\begin{tabular}{|c|c|c|c|c|c|c|c|c|c|}
\hline \multirow{3}{*}{$\begin{array}{l}\text { Dominio } \\
\text { Autopercepción }\end{array}$} & \multicolumn{3}{|c|}{ Primera aplicación $(\mathrm{n}=349)$} & \multicolumn{3}{|c|}{ Segunda aplicación $(\mathrm{n}=176)$} & \multicolumn{3}{|c|}{ Prueba test-retest } \\
\hline & Media \pm DE & Mediana & Rango IC & Media \pm DE & Mediana & Rango IC & Rho_c & & \\
\hline & $20.99 \pm 6.75$ & 22 & 10 & $21.64 \pm 6.58$ & 21.5 & 10 & 0.72 & 0.65 & 0.79 \\
\hline Rol emocional & $19.92 \pm 7.49$ & 20 & 11 & $20.47 \pm 7.19$ & 21 & 11 & 0.71 & 0.63 & 0.78 \\
\hline Rol social & $19.24 \pm 6.74$ & 20 & 10 & $19.87 \pm 6.38$ & 21 & 9 & 0.70 & 0.62 & 0.77 \\
\hline Síntomas de acné & $16.95 \pm 4.91$ & 17 & 7 & $18.44 \pm 5.10$ & 19 & 7 & 0.67 & 0.60 & 0.75 \\
\hline
\end{tabular}




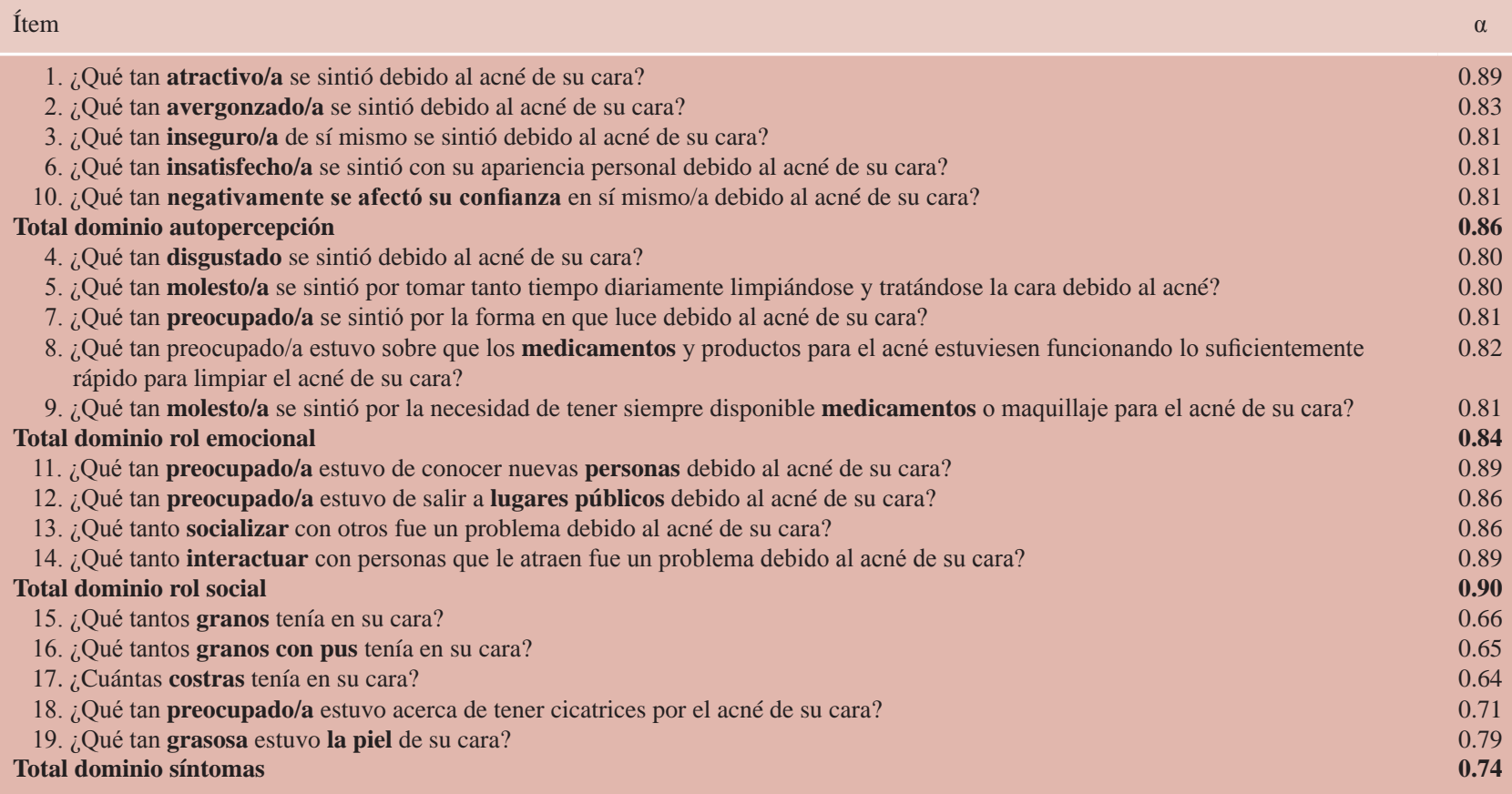

entre las dos aplicaciones. ${ }^{33}$ En nuestro estudio los lapsos para la reaplicación fueron variables y se implementaron dos métodos de evaluación (presencial versus virtual) con el propósito de alcanzar el tamaño de muestra propuesto, estas razones podrían explicar, en alguna medida, las diferencias encontradas entre los estudios. Esta suposición podrá ser probada en estudios futuros que evalúen el lapso de tiempo óptimo de reaplicación o que comparen la concordancia de diferentes métodos de aplicación.

Los resultados de las asociaciones con otras variables con las que se consideró que el constructo de CVrS estaría teóricamente relacionado, constituyen evidencia de la validez de los resultados de la escala, especialmente en la evidencia relacionada con la validez de constructo. Esto se vio reflejado para la mayoría de las asociaciones evaluadas, excepto para el rol emocional y la satisfacción con el estado de salud, pero los resultados obtenidos fueron discretos, lo que es esperable, ya que las variables aunque relacionadas con la $\mathrm{CV}$ rS no están midiendo el mismo concepto. Adicionalmente a pesar que se planteó una hipótesis de no relación entre el rol emocional y la satisfacción con el estado de salud, sí se encontró una asociación negativa; sin embargo, esta asociación fue discreta (cercana a 0).

Los puntajes obtenidos en cada uno de los dominios en la tercera aplicación para evaluar la sensibilidad al cambio fueron más altos en todos los dominios, reflejando un aumento en la $\mathrm{CV}$ rS en cada uno de ellos. Los dominios que presentaron mayor cambio fueron «síntomas de acné» y «autopercepción». Esto se puede relacionar con el resultado de la clasificación de severidad del acné del paciente entre la primera y la tercera aplicación, en la cual más de 59\% reportó una mejoría en la severidad. Estos datos son consistentes con la tendencia reportada previamente por Martin y colegas, quienes encontraron que el Acne-QoL es sensible a los cambios en la severidad del acné facial después de una terapia habitual de tres a cuatro meses. ${ }^{33}$ Con base en estos resultados se puede concluir que la escala es capaz de capturar o detectar variaciones en el tiempo relacionadas con el tratamiento. No obstante, al no contar con grupos de comparación, se requieren investigaciones posteriores para evaluar dicha sensibilidad al cambio en ensayos clínicos aleatorizados.

El análisis factorial confirmatorio indicó que los datos no se ajustan dentro del modelo de cuatro dominios de la versión original en inglés, ${ }^{32,33}$ y a diferencia de los resultados del estudio de Fehnel, ${ }^{34}$ se considera que la estructura factorial propuesta por los autores no se mantiene en esta versión de la escala y en la población en la que se realizó el estudio. La evaluación de la solución factorial más apropiada, empleando un análisis factorial exploratorio de factores principales, mostró que tres factores eran suficientes, y se puede 


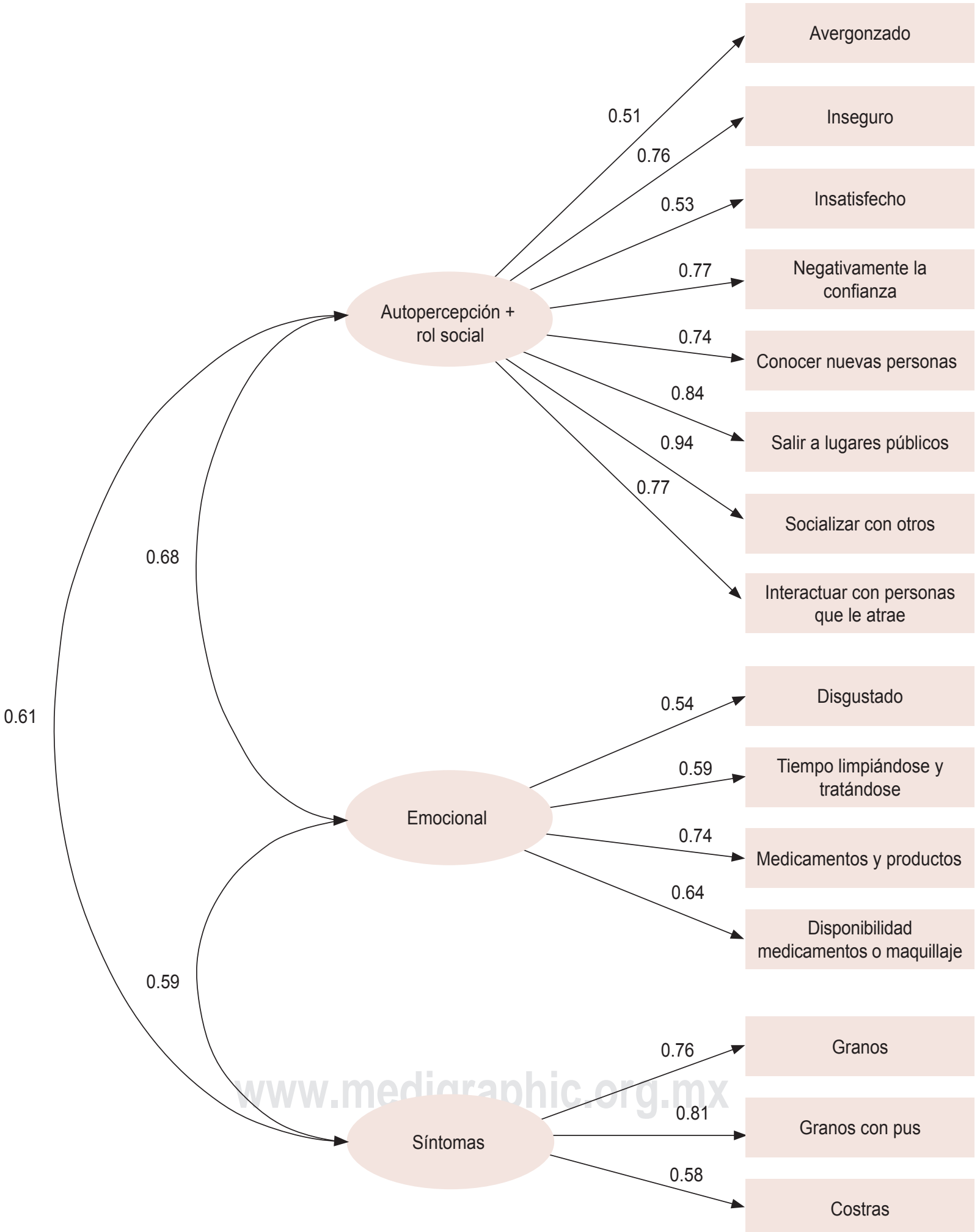

Figura 4: Análisis factorial exploratorio. Método de factores principales - rotación Promax. 
Tabla 5: Sensibilidad al cambio. Tamaño del efecto estandarizado y respuesta media estandarizada (SMR).

\begin{tabular}{|c|c|c|c|c|c|c|}
\hline \multirow[b]{2}{*}{ Subescala (dominio) } & \multirow{2}{*}{$\begin{array}{c}\text { Primera aplicación } \\
\text { Media } \pm \text { DE }\end{array}$} & \multirow{2}{*}{$\begin{array}{c}\text { Tercera aplicación } \\
\text { Media } \pm \text { DE }\end{array}$} & \multirow[b]{2}{*}{ Diferencia media } & \multirow[b]{2}{*}{$\mathrm{DE}$} & \multirow[b]{2}{*}{$\begin{array}{c}\text { Tamaño del } \\
\text { efecto }\end{array}$} & \multirow[b]{2}{*}{$\begin{array}{c}\text { Respuesta media } \\
\text { estandarizada }\end{array}$} \\
\hline & & & & & & \\
\hline Autopercepción & $19.67 \pm 6.62$ & $25.91 \pm 4.07$ & 6.24 & 6.86 & 0.94 & 0.90 \\
\hline Rol emocional & $19.25 \pm 7.79$ & $24.56 \pm 6.43$ & 5.31 & 8.72 & 0.68 & 0.61 \\
\hline Rol social & $17.89 \pm 7.44$ & $22.46 \pm 4.99$ & 4.57 & 6.86 & 0.61 & 0.67 \\
\hline Síntomas de acné & $16.38 \pm 4.68$ & $21.18 \pm 3.86$ & 4.80 & 5.70 & 1.03 & 0.84 \\
\hline
\end{tabular}

concluir que la versión en español de la Acne-QoL presenta una equivalencia semántica y cultural con la versión original en inglés, adecuadas propiedades psicométricas en la muestra evaluada y está compuesta por 15 ítems agrupados en tres dominios únicamente, obteniéndose el primer instrumento específico traducido y validado al español colombiano para la evaluación de la $\mathrm{CVrS}$ en pacientes con acné.

Una limitación de este estudio es que no se puede evaluar de manera directa la validez de criterio, pero se obtuvo evidencia indirecta a partir de evaluar las asociaciones con otras variables con las que se consideró que el constructo de $\mathrm{CVrS}$ en pacientes con acné se encuentra teóricamente relacionado.

Al disponer de un instrumento validado en nuestro medio que permita medir el impacto en la $\mathrm{CV} r \mathrm{~S}$ en los pacientes con acné, se podrá evaluar no sólo el impacto de la enfermedad en nuestros pacientes, sino que se contará con una herramienta que permita evaluar el efecto de las intervenciones teniendo en cuenta la mejoría para el paciente que no necesariamente se relaciona con la mejoría observada por el dermatólogo.

\section{CONCLUSIONES}

Los resultados de este estudio sugieren que la versión en español de la escala Acne-QoL, una escala autoadminis- trada, fácil de aplicar, presentó un proceso de traducción lingüística y adaptación cultural que permite considerarla equivalente conceptual y semánticamente a la versión original en inglés, y que además presenta aceptables características psicométricas en la aplicación realizada en una muestra de pacientes con acné entre 12 y 35 años.

Debido a que la estructura factorial propuesta por los autores de la versión original en inglés no fue confirmada en este estudio, esta escala podría beneficiarse de modificaciones importantes como la eliminación de algunas preguntas, pero esta versión modificada deberá ser probada nuevamente para evaluar sus resultados. Una vez realizados estos ajustes y determinada su nueva estructura factorial sería útil emplear esta escala en la práctica clínica y en investigación para evaluar el impacto del acné y de los tratamientos en la $\mathrm{CVrS}$ de las personas que lo padecen.

\section{BIBLIOGRAFÍA}

1. Rapp SR, Feldman SR, Graham G, Fleischer AB, Brenes G, Dailey M. The Acne Quality of Life Index (Acne-QOLI): development and validation of a brief instrument. $A m \mathrm{~J}$ Clin Dermatol [Internet]. 2006 [cited 2013 May 8]; 7 (3): 185-192. Available from: http://www.ncbi.nlm.nih.gov/ pubmed/16734506

2. Sinclair W, Jordaan HF. Acne guideline 2005 update. S Afr Med J [Internet]. 2005 [cited 2013 May 8]; 95 (11 Pt 2): 881-892. Available from: http://www.ncbi.nlm.nih.gov/pubmed/16344888

3. Dréno B. Assessing quality of life in patients with acne vulgaris: implications for treatment. Am J Clin Dermatol [Internet]. 2006 [cited 2013 May 8]; 7 (2): 99-106. Available from: http://www.ncbi. nlm.nih.gov/pubmed/16605290
4. Thomas DR. Psychosocial effects of acne. J Cutan Med Surg [Internet]. 2004 [cited 2013 Jun 26]; 8 Suppl 4: 3-5. Available from: http://www.ncbi.nlm.nih.gov/pubmed/15778823

5. Behnam B, Taheri R, Ghorbani R, Allameh P. Psychological impairments in the patients with acne. Indian J Dermatol [Internet]. 2013 [cited 2013 Jun 26]; 58 (1): 26-29. Available from: http://www.pubmedcentral.nih.gov/articlerender.fcgi?arti $\mathrm{d}=3555368$ \&tool=pmcentrez\&rendertype=abstract

6. Dreno B, Alirezai M, Auffret N, Beylot C, Chivot M, Daniel F et al. Clinical and psychological correlation in acne: use of the ECLA and CADI scales. Ann Dermatol Venereol [Internet]. 2007 [cited 2013 May 8]; 134 (5 Pt 1): 451-45. Available from: http://www. ncbi.nlm.nih.gov/pubmed/17507843 
7. Layton AM. Psychosocial aspects of acne vulgaris. J Cutan Med Surg [Internet]. 1998 [cited 2013 May 8]; 2 Suppl 3: 19-23. Available from: http://www.ncbi.nlm.nih.gov/pubmed/9873119

8. Motley RJ, Finlay AY. How much disability is caused by acne? Clin Exp Dermatol [Internet]. 1989 [cited 2013 May 8]; 14 (3): 194-198. Available from: http://www.ncbi.nlm.nih.gov/pubmed/2531637

9. Mulder MM, Sigurdsson V, van Zuuren EJ, Klaassen EJ, Faber JA, de Wit JB et al. Psychosocial impact of acne vulgaris. evaluation of the relation between a change in clinical acne severity and psychosocial state. Dermatology [Internet]. 2001 [cited 2013 May 8]; 203 (2): 124-130. Available from: http://www. ncbi.nlm.nih.gov/pubmed/11586010

10. Oakley AM. The Acne Disability Index: usefulness confirmed. Australas J Dermatol [Internet]. 1996 [cited 2013 May 8]; 37 (1): 37-39. Available from: http://www.ncbi.nlm.nih.gov/ pubmed/8936069

11. Pollard WE, Bobbitt RA, Bergner M, Martin DP, Gilson BS. The sickness impact profile: reliability of a health status measure. Med Care [Internet]. 1976 [cited 2013 Jun 26]; 14 (2): 146-155. Available from: http://www.ncbi.nlm.nih.gov/pubmed/1256107

12. Bergner M, Bobbitt RA, Carter WB, Gilson BS. The sickness impact profile: development and final revision of a health status measure. Med Care [Internet]. 1981 [cited 2013 Jun 26]; 19 (8): 787-805. Available from: http://www.ncbi.nlm.nih.gov/ pubmed/7278416

13. Bergner M, Bobbitt RA, Pollard WE, Martin DP, Gilson BS. The sickness impact profile: validation of a health status measure. Med Care [Internet]. 1976 [cited 2013 Jun 26]; 14 (1): 57-67. Available from: http://www.ncbi.nlm.nih.gov/pubmed/950811

14. Alonso J, Anto JM, Moreno C. Spanish version of the Nottingham Health Profile: translation and preliminary validity. Am J Public Health [Internet]. 1990 [cited 2013 Jun 26]; 80 (6): 704-708. Available from: http://www.pubmedcentral.nih.gov/articlerender. fcgi?artid=1404742\&tool=pmcentrez\&rendertype=abstract

15. Wiklund I. The Nottingham Health Profile--a measure of healthrelated quality of life. Scand J Prim Health Care Suppl [Internet]. 1990 [cited 2013 Jun 26]; 1: 15-18. Available from: http://www. ncbi.nlm.nih.gov/pubmed/2100359

16. Parkerson GR, Broadhead WE, Tse CK. The Duke Health Profile. A 17-item measure of health and dysfunction. Med Care [Internet]. 1990 [cited 2013 Jun 26]; 28 (11): 1056-1072. Available from: http://www.ncbi.nlm.nih.gov/pubmed/2250492

17. Parkerson GR, Gehlbach SH, Wagner EH, James SA, Clapp NE, Muhlbaier LH. The Duke-UNC Health Profile: an adult health status instrument for primary care. Med Care [Internet]. 1981 [cited 2013 Jun 26]; 19 (8): 806-828. Available from: http://www. ncbi.nlm.nih.gov/pubmed/7278417

18. Kaplan RM, Ganiats TG, Sieber WJ, Anderson JP. The Quality of Well-Being Scale: critical similarities and differences with SF-36. Int J Qual Health Care [Internet]. 1998 [cited 2013 Jun 26]; 10 (6): 509-520. Available from: http://www.ncbi.nlm.nih. gov/pubmed/9928590

19. Valderas JM, Ferrer M, Mendívil J, Garin O, Rajmil L, Herdman $M$ et al. Development of EMPRO: a tool for the standardized assessment of patient-reported outcome measures. Value Health [Internet]. 2008 [cited 2013 Jun 26]; 11 (4): 700-708. Available from: http://www.valueinhealthjournal.com/article/ S1098-3015(10)60547-5/abstract

20. Assessing health status and quality-of-life instruments: attributes and review criteria. Qual Life Res [Internet]. 2002 [cited 2013 May 8]; 11 (3): 193-205. Available from: http://www.ncbi.nlm. nih.gov/pubmed/12074258
21. Wild D, Grove A, Martin M, Eremenco S, McElroy S, VerjeeLorenz $\mathrm{A}$ et al. Principles of good practice for the translation and cultural adaptation process for patient-reported outcomes (PRO) measures: report of the ISPOR task force for translation and cultural adaptation. Value Health [Internet]. 2005 [cited 2013 Apr 19]; 8 (2): 94-104. Available from: http://www.ncbi.nlm.nih. gov/pubmed/15804318

22. Acquadro C, Conway K, Giroudet C, Mear I. Linguistic validation procedures for original instruments in English. In: Institute MR, editor. Linguistic validation manual for patient-reported outcomes (PRO) instruments. Lyon; 2004.

23. Zigmond AS, Snaith RP. The hospital anxiety and depression scale. Acta Psychiatr Scand [Internet]. 1983 [cited 2013 May 28]; 67 (6): 361-370. Available from: http://www.ncbi.nlm.nih. gov/pubmed/6880820

24. MacCallum RC, Widaman KF, Zhang S, Hong S. Sample size in factor analysis. Psychological Methods. 1999; 4 (1): 84-99.

25. Pett MA, Lackey NR, Sullivan JJ. Making sense of factor analysis: the use of factor analysis for instrument development in health care research [Internet]. SAGE Publications; 2003 [cited 2013 Jun 26]. p. 348. Available from: http://books.google.com/ books?id=9kB5jE2ljS4C\&pgis $=1$

26. Tejero A, Guimera E, Farré JM, Peri JM. Uso clínico del HADS (Hospital Anxiety and Depression Scale) en población psiquiátrica: un estudio de sensibilidad, fiabilidad y validez. Rev Dep Psiquiat Fac Med Barcelona. 1986; 12: 233-238.

27. Hinz A, Finck C, Gómez Y, Daig I, Glaesmer H, Singer S. Anxiety and depression in the general population in Colombia: reference values of the Hospital Anxiety and Depression Scale (HADS). Soc Psychiatry Psychiatr Epidemiol [Internet]. 2014 [cited 2013 Jun 26]; 49 (1): 41-49. Available from: http://www.ncbi.nlm.nih. gov/pubmed/23748887

28. Rico J, Restrepo Forero M, Molina M. Adaptation and validation of the Hospital Anxiety and Depression Scale (HADS) with a sample of Colombian cancer patients form National Institute of Cancer-Colombia. Avances en Medición [Internet]. 2005 [cited 2013 Jun 26]; 3 (1): 73-86. Available from: http://dialnet.unirioja. es/servlet/articulo?codigo=2300105

29. Carver CS, Pozo-Kaderman C, Price AA, Noriega V, Harris SD, Derhagopian RP et al. Concern about aspects of body image and adjustment to early stage breast cancer. Psychosom Med [Internet]. 1998 [cited 2013 Jun 26]; 60 (2): 168-174. Available from: http://www.ncbi.nlm.nih.gov/pubmed/9560865

30. Husted JA, Cook RJ, Farewell VT, Gladman DD. Methods for assessing responsiveness: a critical review and recommendations. J Clin Epidemiol [Internet]. 2000 [cited 2013 May 8]; 53 (5): 459-468. Available from: http://www.ncbi. nlm.nih.gov/pubmed/10812317

31. García M. Sensibilidad al cambio de las medidas de desenlace. Reumatol Clínica [Internet]. 2008 [cited 2013 Jun 26]; 4 (6): 240-247. Available from: http://www.sciencedirect.com/science/ article/pii/S1699258X08755451

32. Girman CJ, Hartmaier S, Thiboutot D, Johnson J, Barber B, DeMuro-Mercon $C$ et al. Evaluating health-related quality of life in patients with facial acne: development of a self-administered questionnaire for clinical trials. Qual Life Res [Internet]. 1996 [cited 2013 Jun 26]; 5 (5): 481-490. Available from: http://www. ncbi.nlm.nih.gov/pubmed/8973127

33. Martin AR, Lookingbill DP, Botek A, Light J, Thiboutot D, Girman CJ. Health-related quality of life among patients with facial acne -- assessment of a new acne-specific questionnaire. Clin Exp 
Dermatol [Internet]. 2001 [cited 2013 May 8]; 26 (5): 380-385. Available from: http://www.ncbi.nlm.nih.gov/ pubmed $/ 11488820$

34. Fehnel SE, McLeod LD, Brandman J, Arbit DI, McLaughlin-Miley CJ, Coombs JH et al. Responsiveness of the Acne-Specific Quality of Life Questionnaire (AcneQoL) to treatment for acne vulgaris in placebo-controlled clinical trials. Qual Life Res [Internet]. 2002 [cited 2013 May 8]; 11 (8): 809-816. Available from: http://www.ncbi. nlm.nih.gov/pubmed/12482164
35. Byrne BM. Adaptation of assessment scales in cross-national research: Issues, guidelines, and caveats. Int Perspect Psychol Res Pract Consult [Internet]. 2016 [cited 2018 Aug 22]; 5 (1): 51-65. Available from: http:// doi.apa.org/getdoi.cfm?doi=10.1037/ipp0000042

36. Tan J, O'Toole A, Zhang X, Dreno B, Poulin Y. Cultural and linguistic validation of acne-QoL in French. J Eur Acad Dermatol Venereol [Internet]. 2012 [cited 2013 Jun 26]; 26 (10): 1310-1314. Available from: http://www.ncbi.nlm.nih.gov/pubmed/21797932

37. Kane M. An argument-based approach to Validity. Psychol Bull. 1992; 112 (3): 527-535. 